\title{
EGFR, HER2 and HER3 expression in esophageal primary tumours and corresponding metastases
}

\author{
QICHUN WEI ${ }^{1,2}$, LIRONG CHEN ${ }^{1}$, LIMING SHENG $^{1}$, HANS NORDGREN $^{3}$, \\ KENNETH WESTER $^{2}$ and JÖRGEN CARLSSON ${ }^{2}$
}

\begin{abstract}
${ }^{1}$ Department of Radiation Oncology, The Second Affiliated Hospital, Cancer Institute, Zhejiang University School of Medicine, Hangzhou 310009, P.R. China; Departments of ${ }^{2}$ Oncology, Radiology and Clinical Immunology, and

${ }^{3}$ Genetics and Pathology, Rudbeck Laboratory, Uppsala University Hospital, SE-751 85 Uppsala, Sweden
\end{abstract}

Received March 12, 2007; Accepted April 30, 2007

\begin{abstract}
The expression of EGFR, HER 2 and HER 3 receptors were analyzed in immunohistochemical preparations from primary esophageal tumours and corresponding lymph node metastases. The goal was to evaluate whether any of these receptors are suitable as targets for radionuclide based imaging and therapy. The receptor expressions were evaluated in parallel samples, primary tumour and metastasis, from each patient $(n=51)$. The majority of the cases were esophageal squamous cell carcinomas, ESCC $(n=40)$. The HercepTest scoring was used for the analysis of both HER2 and EGFR expression $(0,1+, 2+$ or $3+)$. HER3 was only evaluated as negative, weak or strong staining. EGFR overexpression $(2+/ 3+)$ was found in $67.5 \%$ (27/40) of both the ESCC primary tumours and the corresponding lymph node metastases. There were only a few changes in these EGFR-scores: two cases from $2+/ 3+$ to $0 / 1+$ when the primary tumours were compared to the corresponding metastases and 2 changes the other way around. HER 2 overexpression (2+/3+) was found in only 3 of the primary ESCC tumours and 2 of the lymph node metastases. EGFR and HER2 stainings were found mainly in the cell membranes. The HER3 staining (weak or strong) was mainly cytoplasmic and granular and was observed in about half (20/39) of the cases, for both the ESCC primary tumours and the corresponding lymph node metastases. It was concluded that ESCC lymph node metastases generally have a strong expression of EGFR in their cell membranes and to the same extent as in the primary tumours. The stability in EGFR expression is encouraging for efforts to develop radionuclide based EGFR imaging agents. It is also possible that EGFR targeting agents (e.g. Iressa, Tarceva, Erbitux or radiolabelled antibodies) can be applied for therapy of ESCC.
\end{abstract}

Correspondence to: Dr Qichun Wei, Department of Radiation Oncology, The Second Affiliated Hospital, Cancer Institute, Zhejiang University School of Medicine, Hangzhou 310009, P.R. China

E-mail: qichun.wei@bms.uu.se; weiqichun@hotmail.com

Key words: c-erb-B2, esophageal carcinoma, clinical, EGFR, HER2, HER3, metastasis, overexpression of receptors

\section{Introduction}

Cancer of the esophagus is the 6th cause of cancer death worldwide (1). Survival depends on the stage of the disease. Surgical resection has traditionally been considered the gold standard of potentially curative therapy at the early disease stage $(2,3)$. Unfortunately, about $60 \%$ of the patients have unresectable or metastatic disease at the time of diagnosis and/or they are unfit for surgery for medical reasons (4). In this case, chemoradiation is the standard treatment, based on the results of the Radiation Therapy Oncology Group trial 85-01 (5). With advances in surgical techniques, radiation facilities and anti-cancer drugs, the prognosis of esophageal cancer has slowly improved over the past decades. However, the 5-year overall survival rate remains poor, 14\% (6). Metastases and recurrence are the common failure patterns. Additional therapeutical strategies are needed to improve the survival and the quality of life for patients with recurrent and disseminated esophageal cancer. One strategy is receptormediated tumour targeting radionuclide therapy (7), which is based on the delivery of therapeutically relevant radionuclides directly to disseminated tumour cells, with hopefully minimal damage to normal tissues.

The HER family of receptor tyrosine kinases plays a key role in epithelial cellular growth, proliferation, and differentiation. The family consists of four members, the epidermal growth factor receptor (EGFR), HER2, HER3 and HER4 (8). Structurally, all members share common features, including a glycosylated extracellular ligand-binding domain, a hydrophobic transmembrane domain and an intracellular domain with intrinsic protein-tyrosine kinase activity (9). The receptors are usually active in a dimeric form (homo- or heterodimerisation), and interaction between different receptor pairs represents a mechanism for signal diversification and amplification $(10,11)$. EGF and five other ligands can bind to EGFR, and heregulins are the ligands for HER3 and HER4. Since HER2 has no known ligand, and HER3 has no intrinsic tyrosine kinase activity, the signal transduction of HER2 or HER3 is mediated through heterodimerisation with each other or with other receptors in the family.

EGFR overexpression is common in esophageal carcinoma $(12-14)$ and is associated with a poor prognosis $(15,16)$. 
EGFR targeted drugs are clinically available, including smallmolecule tyrosine kinase inhibitors (e.g. Iressa and Tarceva) $(17,18)$, as well as the chimeric monoclonal antibody cetuximab (Erbitux) (19). Kinase domain EGFR mutations have also been found in esophageal carcinomas (20). The frequency of HER2 expression in esophageal carcinoma has been reported to vary from 0 up to about $65 \%$ (21-26). The humanized monoclonal antibody trastuzumab (Herceptin), which specifically targets the extracellular domain of HER2, has boosted the interest of physicians in targeting therapy, as therapeutic benefit was proved in patients with HER2-positive metastatic breast cancer (27). The HER3 expression can be found in normal squamous epithelium of esophagus (28), but so far, the literature on HER3 expression in esophageal carcinoma has been rare (25). The significance of HER4 as a target for cancer therapy is not clear. HER4 has, as EGFR and HER3, ligand stimulated tyrosine kinase activity, but reports differ whether it induces cell division or differentiation (29).

Investigations to characterize possible differences in the expression of these receptors between the primary tumours and metastases are necessary to assess the suitability of receptors as targets in systemic therapy of metastatic esophageal carcinoma. In the present study, the expression of EGFR, HER2 and HER3 was investigated immunohistochemically in both lymph node metastases of esophageal carcinoma and corresponding primary tumours.

\section{Materials and methods}

Patients and samples. Patients with esophageal carcinoma who were diagnosed and treated in the Second Affiliated Hospital, Zhejiang University School of Medicine, between 2002 and 2005, were enrolled in the present study. All of the patients had undergone esophagectomy with lymph node dissection. Paraffin sections from both the primary tumours and the corresponding lymph node metastases were required for inclusion. Tissue samples were not taken from distant metastases, so these were not available for analysis. Patients who had received preoperative radiotherapy or preoperative chemotherapy were excluded. Totally, 51 patients with high quality material of both primary tumours and corresponding metastases were finally included in the study. The patient and tumour characteristics of the analyzed patients are shown in Table I.

Briefly, the tissues were fixed in $4 \%$ buffered formalin, dehydrated and embedded in paraffin. Sections, $4-\mu \mathrm{m}$ thick, were then cut and deparaffinized in xylene and hydrated through graded concentrations of ethanol to distilled water.

EGFR-staining. EGFR was assessed by immunohistochemistry using a streptavidin-biotin complex technique as previously described (30). After deparaffinization of the sections, endogenous peroxidase was blocked in $0.3 \% \mathrm{H}_{2} \mathrm{O}_{2}$ in PBS for $20 \mathrm{~min}$. Then, enzymatic antigen retrieval was done in $0.05 \%$ protease K (Code no. S3020, Dako, Glostrup, Denmark) in PBS for 10 min at room temperature. The slides were preincubated in PBS for $10 \mathrm{~min}$. The primary mouse monoclonal antibody directed against the EGF receptor (clone 31G7, Zymed labs, South San Francisco, CA) was diluted 1:100 and incubated overnight at $4{ }^{\circ} \mathrm{C}$. The secondary biotinylated antibodies (goat anti-mouse from Dako) and the
Table I. Tumour and patient characteristics $(n=51)$.

Characteristics

No. of patients (\%)

\begin{tabular}{lc}
\hline Tumour type & \\
Esophageal, squamous carcinomas & $40(78)$ \\
Esophageal, adenocarcinomas & $6(12)$ \\
Small cell tumours & $3(6)$ \\
Neuroendocrine tumour & $1(2)$ \\
Carcinosarcoma & $1(2)$
\end{tabular}

Differentiation of the

squamous carcinomas

Low

Moderate

$20(50)$

High

$\mathrm{T}$-stages of the

squamous carcinomas

$\mathrm{T} 2$

$\mathrm{T} 3$

$\mathrm{T} 4$

Mean length (mm),

$61 \pm 22$

squamous carcinomas

Average age (years),

$58 \pm 10$

Table II. EGFR-scores for the analyzed primary esophageal squamous cell carcinoma and the corresponding lymph node metastases $(n=40)$.

\begin{tabular}{lcccr}
\hline $\begin{array}{l}\text { Primary tumour } \\
\text { EGFR-scores }\end{array}$ & \multicolumn{4}{c}{ Lymph node metastases EGFR-scores } \\
\cline { 2 - 5 } & 0 & $1+$ & $2+$ & $3+$ \\
\hline 0 & 5 & 0 & 1 & 0 \\
$1+$ & 3 & 3 & 0 & 1 \\
$2+$ & 1 & 0 & 2 & 1 \\
$3+$ & 0 & 1 & 5 & 17
\end{tabular}

The scoring was based on a scale where 0 corresponded to completely negative staining, $1+$ corresponded to faint perceptible staining of the tumour cell membranes, $2+$ corresponded to moderate staining of the entire tumour cell membranes and $3+$ was strong circumferential staining of the entire tumour cell membranes creating a fishnet pattern.

peroxidase-labelled streptavidin-biotin complex (Dako) were diluted 1:200 and incubated for $30 \mathrm{~min}$ at room temperature. All slides were developed in $0.05 \%$ diamino benzidine (Sigma, St. Louis, MO) for $5 \mathrm{~min}$ and counterstained in Harris haematoxylin (Sigma). Finally, the slides were dehydrated through graded alcohol to xylene and mounted in organic mounting medium (Pertex ${ }^{\circledR}$, Histolab, Gothenburg, Sweden). 
Table III. Major results from the EGFR-scores analyses of esophageal squamous cell carcinoma $(n=40)$.

\begin{tabular}{lcr}
\hline EGFR-scores characteristics & Fraction & $\%$ \\
\hline Primary tumours with 3+ & $23 / 40$ & 57.5 \\
Primary tumours with 2+ or 3+ & $27 / 40$ & 67.5 \\
Lymph node metastases with 3+ & $19 / 40$ & 47.5 \\
Lymph node metastases with 2+ or 3+ & $27 / 40$ & 67.5 \\
Unchanged EGFR-scores in lymph node metastases vs. the primary tumour & $27 / 40$ & 67.5 \\
Changed EGFR-scores in lymph node metastases vs. the primary tumour & $13 / 40$ & 32.5 \\
Patients who had 0 or 1+ in primary tumours and changed to 2+ or 3+ in lymph node metastases & $2 / 40$ & 5 \\
Patients who had 2+ or 3+ in primary tumours and changed to 0 or 1+ in lymph node metastases & $2 / 40$ & 5 \\
\hline
\end{tabular}

HER2-staining. The HER2 immunohistochemical staining was made as described earlier (31). After deparaffinization, the sections were incubated in methanol and hydrogen peroxide for $30 \mathrm{~min}$ in order to quench endogenous peroxidase. Antigen retrieval was done in waterbath at $95-98^{\circ} \mathrm{C}, \mathrm{pH} 6.0$ for $40 \mathrm{~min}$. Thereafter the glasses were cooled at room temperature and then washed in distilled water. Immunohistochemical staining was performed using the Elite ABC Kit (Vectastain, Vector Laboratories, Burlingame, CA). Blocking serum was applied for $15 \mathrm{~min}$ and followed by incubation with rabbit anti-human c-erbB-2 oncoprotein (code No. A 0485, Dako) diluted 1:350. Sections were then incubated with the biotinylated secondary antibody and were visualised by using the peroxidase substrate 3-amino-9-ethyl-carbazole (AEC) (Sigma A-5754) as chromogen. Finally, the sections were counterstained with Mayer's haematoxylin and mounted with Aquamount (BDH Ltd., Poole, UK).

HER3-staining. After deparaffinization, the sections were incubated in methanol and hydrogen peroxide for $30 \mathrm{~min}$ in order to quench endogenous peroxidase. Antigen retrieval was done in a pressure chamber at $125^{\circ} \mathrm{C}, \mathrm{pH} 9.0$ for $4 \mathrm{~min}$. Thereafter the glasses were cooled at room temperature and then washed in distilled water. Immunohistochemical staining was performed using the Elite ABC Kit (Vectastain, Vector Laboratories). Blocking serum was applied for $15 \mathrm{~min}$ and followed by incubation with the monoclonal antibody MAB4021 (Chemicon, Temecula, CA) diluted 1:1,000. Sections were then incubated with a biotinylated secondary antibody and visualised by using the peroxidase substrate 3amino-9-ethyl-carbazole (AEC) (Sigma A-5754) as chromogen. Finally, the sections were counterstained with Mayer's haematoxylin and mounted with Aquamount (BDH Ltd.).

EGFR and HER2-scores. The HER2 expression was scored using the HercepTest criterion. The HER2-score was based on a scale where 0 corresponded to tumour cells that were completely negative, $1+$ corresponded to faint perceptible staining of the tumour cell membranes, 2+ corresponded to moderate staining of the entire tumour cell membranes and $3+$ was strong circumferential staining of the entire tumour cell membranes creating a fishnet pattern. The Canadian and the Dako HercepTest guidelines (32) were applied, which require $>10 \%$ of the tumour cells to be stained. Cytoplasmic staining was considered non-specific and was not included in the scoring. As positive controls we used in house positive tissue sections as well as positive sections supplied by Dako. As negative controls we used normal tissues, which are expected not to express HER2, such as connective tissue seen in the same sections as the tumour cells. In the metastases sections we used lymphocytes and the surrounding capsule of the lymph nodes as negative internal controls. The expression pattern of EGFR is quite similar to that of HER2, and EGFR expression was therefore evaluated using the same scoring criterion as for HER2. As EGFR positive controls we used in house positive control skin tissue sections. As negative controls we used connective tissue seen in the same sections as the tumour cells. In the metastases sections we used lymphocytes and the surrounding capsule of the lymph nodes as negative internal controls.

HER3 evaluation. The HER3 staining was evaluated as negative, weak, and strong staining. Negative corresponded to tumour cells that were not at all stained, weak corresponded to faint staining of the tumour cytoplasm with/without stained granules, and strong corresponded to intensive tumour granular cytoplasmic staining. As positive controls we used normal esophageal epithelium staining, as seen in our sections (internal controls). Such positive reference staining of normal esophageal epithelium can also be found at the website: www.proteinatlas. org. As negative controls we used tumour stroma of connective tissue character and non-tumour invaded areas of lymph nodes in our sections (internal controls).

Excluded patient cases. In two cases, no tumour cells could be found in the sections of lymph nodes. In another case, there were no tumour cells in the sections supposed to be primary esophageal cancer. In addition, one patient was diagnosed with esophageal cancer, but it turned out to be a gastric cancer invading the esophagus. Thus, the initial 55 patient cases were reduced to 51 cases with representative material from both primary tumours and the corresponding metastases.

\section{Results}

Expression of EGFR. The EGFR-scores for the analyzed 40 primary squamous cell carcinoma and the corresponding 

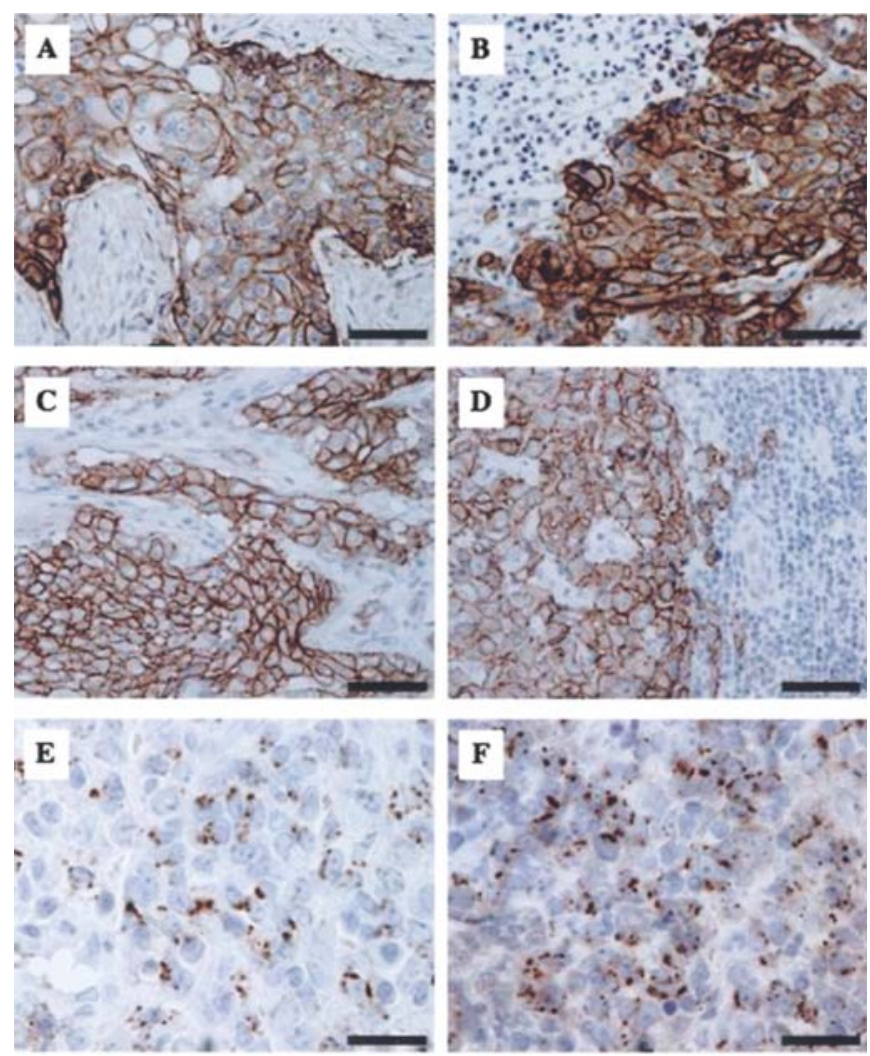

Figure 1. Comparisons of the immunohistochemical brown receptor stainings of primary esophageal squamous cell carcinomas (A, C and E) and corresponding metastases (B, D and $\mathrm{F}$ ) from 3 patients. The EGFR-stained examples (A and B) scored 3+, the HER2-stained examples (C and D) scored 3+ and the HER3-stained examples (E and F) scored S. Nuclear stainings (weak blue) were made with haematoxylin. The bars in A-D correspond to $50 \mu \mathrm{m}$ (micrographs taken with objective $\mathrm{x} 40$ ) and the bars in $\mathrm{E}$ and $\mathrm{F}$ correspond to $20 \mu \mathrm{m}$ (micrographs taken with objective $\mathrm{x} 100$ ).

40 lymph node metastases are shown in Table II. EGFR overexpression $(2+$ or $3+$ ) was found in $67.5 \%(27 / 40)$ of the ESCC primary tumours and of the corresponding lymph node metastases. There was good agreement between the primary tumours and the corresponding lymph node metastases in the majority of cases. The major results from the EGFR-score analyses are summarized in Table III. Thirteen changes were observed. However, there were only two patients who had $2+$ or $3+$ in the primary tumours and changed to 0 or $1+$ in lymph node metastases, and another two patients who had 0 or $1+$ in the primary tumours and changed to $2+$ or $3+$ in lymph node metastases. Examples of staining patterns for a primary tumour and the corresponding metastasis (which both were scored as 3+) are shown in Fig. 1A and B.

Two of the 6 cases of esophageal adenocarcinoma had 2+ EGFR-scores, one case had 1+ EGFR-score, and the other 3 cases had no EGFR staining. There were no changes of EGFR-scores between primary adenocarcinoma and the corresponding lymph node metastases.

Expression of HER2. Table IV shows the HER2-scores for the analyzed primary squamous cell carcinoma and the corresponding lymph node metastases. In one case, the tissue sections of the lymph node metastases were washed away during the staining procedure, so these samples were excluded.
Table IV. HER2-scores for the analyzed primary esophageal squamous cell carcinoma and the corresponding lymph node metastases $(n=39)$.

\begin{tabular}{lcccr}
\hline \multirow{2}{*}{$\begin{array}{l}\text { Primary tumour } \\
\text { HER2-scores }\end{array}$} & \multicolumn{4}{c}{ Lymph node metastases HER2-score } \\
\cline { 2 - 5 } & 0 & $1+$ & $2+$ & $3+$ \\
\hline 0 & 27 & 1 & 0 & 0 \\
$1+$ & 3 & 4 & 1 & 0 \\
$2+$ & 1 & 1 & 0 & 0 \\
$3+$ & 0 & 0 & 0 & 1 \\
\hline
\end{tabular}

The scoring was based on a scale where 0 corresponded to completely negative staining, $1+$ corresponded to faint perceptible staining of the tumour cell membranes, $2+$ corresponded to moderate staining of the entire tumour cell membranes and $3+$ was strong circumferential staining of the entire tumour cell membranes creating a fishnet pattern.

Table V. HER3-scores for the analyzed primary esophageal squamous cell carcinoma and the corresponding lymph node metastases $(n=39)$.

\begin{tabular}{lccr}
\hline Primary tumour & \multicolumn{3}{c}{ Lymph node metastases HER3-scores } \\
\cline { 2 - 4 } & 0 & W & S \\
\hline 0 & 19 & 1 & 0 \\
W & 1 & 14 & 1 \\
S & 0 & 0 & 3 \\
\hline
\end{tabular}

0 , no stained cells; W, weak cellular staining of cytoplasm with or without weak stained granules; $\mathrm{S}$, strong granular cytoplasmic staining.

Thus, 39 cases were analyzed. Generally, HER2 had lower expression than EGFR. Overexpression $(2+$ or $3+)$ of HER2 was only found in 3 of the primary ESCC tumours and 2 of the lymph node metastases. Twenty-six of the 39 cases had no HER2 staining either in the primary tumour or in the corresponding lymph node metastases. Only one case of these patients showed $3+$ in the primary tumour, and this case showed $3+$ also in the corresponding lymph node metastasis (Fig. 1C and D). In total, 7 changes were observed. Two metastases had a higher score compared to the corresponding primary tumour, and five metastases had a lower score compared to the corresponding primary tumour.

Out of the 6 cases of esophageal adenocarcinoma, one case had 2+ HER2-score, and the other 5 cases had no HER2 staining. In all these cases, no changes of HER2scores between primary adenocarcinoma and the corresponding lymph node metastases were observed.

Expression of HER3. The HER3-scores for the analyzed primary esophageal squamous cell carcinoma and the corresponding lymph node are shown in Table V. No tissue sections were available in one case, so totally 39 cases were 
analysed. Strong staining of HER3 was found in $3(8 \%)$ of the primary ESCC tumours and the lymph node metastases (Fig. IE and F) and weak staining in 15 (38\%) of the cases. Nineteen of the 39 cases had no HER3 staining either in the primary tumour or in the corresponding lymph node metastases. Good agreement between HER3 expression of the primary tumours and the corresponding lymph node metastases was observed in the majority of cases. There were only small changes: 2 metastases had a higher score compared to the corresponding primary tumour, and only one had a lower score compared to the corresponding primary tumour.

As to the 6 cases of esophageal adenocarcinoma, 2 cases had strong staining, two had weak staining, and the other 2 had negative staining. In all these cases, there was good concordance between primary adenocarcinoma and the corresponding lymph node metastases.

Rare cases. The receptor expression in a few rare types of esophageal tumours (three small cell cancers, one carcinosarcoma and one neuroendocrine carcinoma) was negative for all three receptors, both in the primary tumours and in the lymph node metastases.

\section{Discussion}

The expression of epidermal growth factor receptor, EGFR, has been studied in primary esophageal cancers, and overexpression is common (12-14) and associated with a poor prognosis $(15,16)$. EGFR targeted drugs are now commercially available, including small-molecule tyrosine kinase inhibitors (e.g. Iressa and Tarceva) $(17,18)$, as well as the chimeric monoclonal antibody cetuximab (Erbitux) (19). However, with the exception of Iressa, these have not yet been tried for therapy of esophageal cancers. Iressa has been used as second-line treatment of advanced esophageal cancer patients in one clinical trial showing limited success (33). Based on previous studies, it is unclear whether the metastases lose, gain, or retain EGFR status relative to the primary tumour. Studies on the EGFR status of metastatic lymph nodes of esophageal cancer will provide precious knowledge to evaluate whether the receptor is of interest for diagnostic and/or therapeutic procedures or not.

In the present study, EGFR expression was identified in the primary esophageal carcinoma (essentially of squamous carcinoma type, ESCC) and corresponding lymph node metastases. According to the recently published data, EGFR overexpression ranges from 50 to $80 \%$ (12-14,25), and our result, $67.5 \%$, is consistent with these findings. Furthermore, we found that the frequency of EGFR overexpression $(2+/ 3+)$ in lymph node metastases was as high as in the ESCC primary tumours. Only 2 patients (5\%) with EGFR overexpression in the primary tumour had lower EGFR scores in the corresponding lymph node metastases. Moreover, in another 2 patients, EGFR overexpression was gained in lymph node metastases, while the primary tumours had low scores.

One third of esophageal adenocarcinoma cases had EGFR overexpression. No changes of EGFR-scores between primary tumours and the corresponding lymph node metastases were observed for the adenocarcinomas.
To our knowledge, the question of EGFR status in lymph node metastases versus primary esophageal cancer has not been addressed. An exception is a study by Itakura et al (34) who reported results from 46 cases esophageal carcinoma with lymph node metastases. The EGFR immunostaining of lymph nodes was classified as positive or negative, and they found that $88 \%$ of the metastatic lymph nodes were EGFR positive. In the cases with EGFR expression in the primary tumours, $94.3 \%$ of the lymph node metastases were EGFR positive. Our results, together with those of Itakura et al, indicate that EGFR expression is stable when comparing the lymph node metastases with the primary esophageal cancer. It seems that EGFR expression in the primary tumours, which can readily be determined after surgery or biopsy, can predict EGFR-positive lymph node metastases with a reasonably high probability.

The expression of HER2 in our samples was not so common as compared to that of EGFR. Overexpression $(2+/ 3+)$ of HER2 was found only in $7.7 \%$ of the primary ESCC tumours and only in 1 of the 6 cases of esophageal adenocarcinoma. A literature review shows an obvious controversy regarding HER2 expression in esophageal carcinoma. Gibault et al (12), Lam et al (21) and Reichelt et al (26) found high HER2 expression in only $2.8 \%, 10 \%$ and $7 \%$, respectively, of the studied ESCC. However, Akamatsu et al (23) and Friess et al (25) reported HER2 overexpression in more than half of the studied cases. There might be many reasons for the observed differences, including patient selection, methodology of the immunohistochemical procedures, scoring and the definition of overexpression.

We have only found 2 studies in the literature concerning the stability of HER2 expression between primary esophageal tumours and the corresponding lymph node metastases $(22,26)$. In the study by Mimura et al $(22)$ only 3 cases with HER 2 expression, scored as $3+$, of 66 primary ESCC tumours. In these 3 cases, HER2 overexpression was preserved in the metastatic lymph nodes. In 6 cases with $2+$ in the primary tumours, 4 cases had metastases that were analysed, and only 2 out of the 4 lymph node metastases retained HER 2 expression as $2+$. The other 2 had negative HER2 staining. In the study by Reichelt et al (26) there was a reasonably good agreement between the HER2 expression in the primary tumours and the corresponding metastases. In our study, the only case with $3+$ in the primary tumour also had $3+$ in the corresponding lymph node. For the 2 cases with $2+$ in the primary tumour, lower HER2 scores were seen in the lymph node metastases.

Thus, the frequency of HER2 overexpression in esophageal cancer seems to be low, which suggests a limited role of this receptor as a target in the treatment of esophageal cancer. However, for the patients with strong membrane staining (3+) in the primary tumour, the same HER2 expression pattern in the lymph node metastases could be expected, which might be of interest for HER2 targeted therapy in those few cases.

The staining pattern of HER3 in tumours is not fully understood. Some authors reported mainly cytoplasmic staining of HER3 in esophageal, ovarian, lung, and breast cancer $(25,35,36)$, while cytoplasmic and membrane staining has been reported for colorectal carcinomas (37). So far, there are no commonly accepted scoring criteria for HER3 evaluation. 
In our study, HER3 staining was restricted to the cytoplasm, exhibiting diffuse and/or granular cytoplasmic staining. HER3 expression was observed in about half $(19 / 39, \approx 49 \%)$ of the ESCC primary tumours and the corresponding lymph node metastases, and $16 \%$ of the positive cases had strong staining. For esophageal adenocarcinoma, two thirds had positive HER3 staining and half of them had strong staining. This should be compared with previous reports on HER3 staining in various cancers. Positive HER3 staining has been reported in about $64 \%$ of the studied esophageal cancers $(25)$, in $45-55 \%$ of ovarian cancers $(35,36)$ and about 17 $30 \%$ of colorectal carcinomas (37). Since HER3 staining is mainly cytoplasmic, it seems that this receptor is, at least for esophageal carcinomas, of low interest to be a target for radionuclide therapy using macromolecules.

Five rarely seen cases of esophageal tumours with other histological types than squamous cell carcinoma and adenocarcinoma were evaluated in this study, including 3 cases of small cell carcinoma, one case of carcinosarcoma and one case of neuroendocrine carcinoma. The EGFR, HER2, and HER3 receptor expression in these cases was negative in both the primary tumours and the lymph node metastases. It could, as a comparison, be noted that EGFR and HER2 are expressed to some extent in small cell lung cancer $(38,39)$ and uterine endometrial carcinosarcoma (40).

As mentioned in the introduction, additional strategies are needed to improve the survival and the quality of life for patients with recurrent and disseminated esophageal cancer. Targeted therapy with antibodies or small-molecule tyrosine kinase inhibitors has been proved to be a promising therapy strategy in lymphoma, lung, colorectal and breast cancer $(41,42)$ and could possibly also be so for esophageal cancer. Receptor mediated tumour targeted radionuclide therapy could be another strategy (7). This strategy is based on delivery of therapeutically relevant radionuclides to disseminated tumour cells with hopefully minimal damage to normal tissues.

For a receptor to be of interest for targeting, similar expression in both the primary tumours and the disseminated lesions is required. The stability in EGFR expression in esophageal cancer is encouraging for efforts to use EGFR targeting agents (e.g. Iressa, Tarceva, Erbitux or radiolabelled antibodies) for therapy of ESCC. For individual esophageal cancer patients with HER2 overexpression, antiHER2 agents (trastuzumab, pertuzumab or radiolabelled antibodies) could possibly also be used.

\section{Acknowledgments}

The authors thank Ulrika A. Larsson for help with the immunohistochemical stainings. The authors acknowledge economical support by a grant from the Swedish Cancer Research Society and a grant from the National Natural Science Foundation of China.

\section{References}

1. Tew WP, Kelsen DP and Ilson DH: Targeted therapies for esophageal cancer. Oncologist 10: 590-601, 2005.

2. Daly JM, Fry WA, Little AG, Winchester DP, McKee RF, Stewart AK and Fremgen AM: Esophageal cancer: results of an American College of Surgeons Patient Care Evaluation Study. J Am Coll Surg 190: 562-572, 2000.
3. Van Lanschot JJ, Aleman BM and Richel DJ: Esophageal carcinoma: surgery, radiotherapy, and chemotherapy. Curr Opin Gastroenterol 18: 490-495, 2002.

4. Crehange G, Bosset M, Fabrice L, Buffet-Miny J, Dumas JL, Mercier M, Puyraveau M, Maingon P and Bosset JF: Tumor volume as outcome determinant in patients treated with chemoradiation for locally advanced esophageal cancer. Am J Clin Oncol 29: 583-587, 2006.

5. Herskovic A, Martz K, Al-Sarraf M, Leichman L, Brindle J, Vaitkevicius V, Cooper J, Byhardt R, Davis L and Emami B: Combined chemotherapy and radiotherapy compared with radiotherapy alone in patients with cancer of the esophagus. N Eng J Med 326: 1593-1598, 1992 .

6. Enzinger PC and Mayer RJ: Esophageal cancer. N Engl J Med 349: 2241-2252, 2003.

7. Carlsson J, Forssell Aronsson E, Hietala SO, Stigbrand T and Tennvall J: Tumour therapy with radionuclides: assessment of progress and problems. Radiother Oncol 66: 107-117, 2003.

8. Citri A and Yarden Y: EGF-ERBB signalling: towards the systems level. Nat Rev Mol Cell Biol 7: 505-516, 2006.

9. Yarden Y and Ullrich A: Growth factor receptor tyrosine kinases. Ann Rev Biochem 57: 443-478, 1988.

10. Yarden Y and Sliwkowski MX: Untangling the ErbB signalling network. Nat Rev Mol Cell Biol 2: 127-137, 2001.

11. Memon AA, Sorensen BS, Meldgaard P, Fokdal L, Thykjaer T and Nexo E: The relation between survival and expression of HER 1 and HER 2 depends on the expression of HER 3 and HER4: a study in bladder cancer patients. Br J Cancer 94: 1703-1709, 2006.

12. Gibault L, Metges JP, Conan-Charlet V, Lozac'h P, Robaszkiewicz M, Bessaguet C, Lagarde N and Volant A: Diffuse EGFR staining is associated with reduced overall survival in locally advanced oesophageal squamous cell cancer. Br J Cancer 93: 107-115, 2005.

13. Hanawa M, Suzuki S, Dobashi Y, Yamane T, Kono K, Enomoto N and Ooi A: EGFR protein overexpression and gene amplification in squamous cell carcinomas of the esophagus. Int $\mathbf{J}$ Cancer 118: 1173-1180, 2006.

14. Sunpaweravong P, Sunpaweravong S, Puttawibul P, Mitarnun W, Zeng C, Baron AE, Franklin W, Said S and Varella-Garcia M: Epidermal growth factor receptor and cyclin D1 are independently amplified and overexpressed in esophageal squamous cell carcinoma. J Cancer Res Clin Oncol 131: 111-119, 2005.

15. Aloia TA, Harpole DH Jr, Reed CE, Allegra C, Moore MB, Herndon JE II and D'Amico TA: Tumor marker expression is predictive of survival in patients with esophageal cancer. Ann Thorac Surg 72: 859-866, 2001.

16. Gibson MK, Abraham SC, Wu TT, Burtness B, Heitmiller RF, Heath $\mathrm{E}$ and Forastiere A: Epidermal growth factor receptor, p53 mutation, and pathological response predict survival in patients with locally advanced esophageal cancer treated with preoperative chemoradiotherapy. Clin Cancer Res 9: 6461-6468, 2003.

17. Ciardiello F, Caputo R, Bianco R, Damiano V, Pomatico G, De Placido S, Bianco AR and Tortora G: Antitumor effect and potentiation of cytotoxic drugs activity in human cancer cells by ZD-1839 (Iressa), an epidermal growth factor receptor-selective tyrosine kinase inhibitor. Clin Cancer Res 6: 2053-2063, 2000.

18. Hightower M: Erlotinib (OSI-774, Tarceva), a selective epidermal growth factor receptor tyrosine kinase inhibitor, in combination with chemotherapy for advanced non-small cell lung cancer. Clin Lung Cancer 4: 336-338, 2003.

19. Goldberg RM: Cetuximab. Nat Rev Drug Discov Suppl: S10-S11, 2005.

20. Kwak EL, Jankowski J, Thayer SP, Lauwers GY, Brannigan BW, Harris PL, Okimoto RA, Haserlat SM, Driscoll DR, Ferry D, Muir B, Settleman J, Fuchs CS, Kulke MH, Ryan DP, Clark JW, Sgroi DC, Haber DA and Bell DW: Epidermal growth factor receptor kinase domain mutations in esophageal and pancreatic adenocarcinomas. Clin Cancer Res 12: 4283-4287, 2006.

21. Lam KY, Tin L and Ma L: C-erbB-2 protein expression in oesophageal squamous epithelium from oesophageal squamous cell carcinomas, with special reference to histological grade of carcinoma and pre-invasive lesions. Eur J Surg Oncol 24: 431-435, 1998.

22. Mimura K, Kono K, Hanawa M, Mitsui F, Sugai H, Miyagawa N, Ooi A and Fujii H: Frequencies of HER-2/neu expression and gene amplification in patients with oesophageal squamous cell carcinoma. Br J Cancer 92: 1253-1260, 2005. 
23. Akamatsu M, Matsumoto T, Oka K, Yamasaki S, Sonoue H, Kajiyama Y, Tsurumaru M and Sasai K: c-erbB-2 oncoprotein expression related to chemoradioresistance in esophageal squamous cell carcinoma. Int J Radiat Oncol Biol Phys 57: 1323-1327, 2003 .

24. Dreilich M, Wanders A, Brattstrom D, Bergstrom S, Hesselius P, Wagenius G and Bergqvist M: HER-2 overexpression (3+) in patients with squamous cell esophageal carcinoma correlates with poorer survival. Dis Esophagus 19: 224-231, 2006.

25. Friess H, Fukuda A, Tang WH, Eichenberger A, Furlan N, Zimmermann A, Korc M and Buchler MW: Concomitant analysis of the epidermal growth factor receptor family in esophageal cancer: overexpression of epidermal growth factor receptor mRNA but not of c-erbB-2 and c-erbB-3. World J Surg 23: 1010-1018, 1999.

26. Reichelt U, Duesedau P, Tsourlakis MCh, Quaas A, Link BC, Schurr PG, Kaifi JT, Gros SJ, Yekebas EF, Marx A, Simon R, Izbicki JR and Sauter G: Frequent homogeneous HER-2 amplification in primary and metastatic adenocarcinoma of the esophagus. Mod Pathol 20: 120-129, 2007.

27. Barnes DM and Miles DW: Response of metastatic breast cancer to trastuzumab? Lancet 355: 160-161, 2000.

28. Prigent SA, Lemoine NR, Hughes CM, Plowman GD, Selden C and Gullick WJ: Expression of the c-erbB-3 protein in normal human adult and fetal tissues. Oncogene 7: 1273-1278, 1992.

29. Gullick WJ: c-erbB-4/HER4: friend or foe? J Pathol 200: 279-281, 2003.

30. Wester K, Sjostrom A, De la Torre M, Carlsson J and Malmstrom PU: HER-2-a possible target for therapy of metastatic urinary bladder carcinoma. Acta Oncol 41: 282-288, 2002.

31. Carlsson J, Nordgren H, Sjostrom J, Wester K, Villman K, Bengtsson NO, Ostenstad B, Lundqvist $\mathrm{H}$ and Blomqvist C: HER 2 expression in breast cancer primary tumours and corresponding metastases. Original data and literature review. Br J Cancer 90: 2344-2348, 2004.

32. Bilous M, Dowsett M, Hanna W, Isola J, Lebeau A, Moreno A, Penault-Llorca F, Rüschoff J, Tomasic G and van de Vijver M: Current perspectives on HER2 testing: a review of National Testing Guidelines. Mod Pathol 16: 173-182, 2003.

33. Janmaat ML, Gallegos-Ruiz MI, Rodriguez JA, Meijer GA, Vervenne WL, Richel DJ, van Groeningen C and Giaccone G: Predictive factors for outcome in a phase II study of gefitinib in second-line treatment of advanced esophageal cancer patients. J Clin Oncol 24: 1612-1619, 2006.
34. Itakura Y, Sasano H, Shiga C, Furukawa Y, Shiga K, Mori S and Nagura $\mathrm{H}$ : Epidermal growth factor receptor overexpression in esophageal carcinoma. An immunohistochemical study correlated with clinicopathologic findings and DNA amplification. Cancer 74: 795-804, 1994.

35. Tanner B, Hasenclever D, Stern K, Schormann W, Bezler M, Hermes M, Brulport M, Bauer A, Schiffer IB, Gebhard S, Schmidt M, Steiner E, Sehouli J, Edelmann J, Lauter J, Lessig R, Krishnamurthi K, Ullrich A and Hengstler JG: ErbB-3 predicts survival in ovarian cancer. J Clin Oncol 24: 4317-4323, 2006.

36. Leibl S, Bodo K, Gogg-Kammerer M, Hrzenjak A, Petru E, Winter R, Denk H and Moinfar F: Ovarian granulosa cell tumors frequently express EGFR (Her-1), Her-3, and Her-4: an immunohistochemical study. Gynecol Oncol 101: 18-23, 2006.

37. Kountourakis P, Pavlakis K, Psyrri A, Rontogianni D, Xiros N, Patsouris E, Pectasides D and Economopoulos T: Prognostic significance of HER3 and HER4 protein expression in colorectal adenocarcinomas. BMC Cancer 6: 46, 2006.

38. Micke P, Hengstler JG, Ros R, Bittinger F, Metz T, Gebhard S, Beeh KM, Oesch F and Buhl R: c-erbB-2 expression in smallcell lung cancer is associated with poor prognosis. Int J Cancer 92: 474-479, 2001.

39. Damstrup L, Rygaard K, Spang-Thomsen M and Poulsen HS: Expression of the epidermal growth factor receptor in human small cell lung cancer cell lines. Cancer Res 52: 3089-3093, 1992.

40. Livasy CA, Reading FC, Moore DT, Boggess JF and Lininger RA: EGFR expression and HER2/neu overexpression/amplification in endometrial carcinosarcoma. Gynecol Oncol 100: 101-106, 2006.

41. Mendelsohn $\mathbf{J}$ and Baselga J: Epidermal growth factor receptor targeting in cancer. Semin Oncol 33: 369-385, 2006.

42. Piccart-Gebhart MJ, Procter M, Leyland-Jones B, Goldhirsch A, Untch M, Smith I, Gianni L, Baselga J, Bell R, Jackisch C, Cameron D, Dowsett M, Barrios CH, Steger G, Huang CS, Andersson M, Inbar M, Lichinitser M, Lang I, Nitz U, Iwata H, Thomssen C, Lohrisch C, Suter TM, Ruschoff J, Suto T, Greatorex V, Ward C, Straehle C, McFadden E, Dolci MS, Gelber RD and Herceptin Adjuvant (HERA) Trial Study Team: Trastuzumab after adjuvant chemotherapy in HER2-positive breast cancer. N Engl J Med 353: 1659-1672, 2005. 